\title{
La legge della memoria
}

Fabio Caffarena ${ }^{1}$

Between 1948 and 1949, a furniture-polisher from Rieti incarcerated in Pordenone Prison writes a peculiar autobiographical memoir, a self-defence for the local courthouse in which he denies the accusations of creating a false identity and stealing. These crimes - he submits - were caused by the exceptional circumstances in which he found himself after 8 September 1943, when he escaped from the prison of Finale Ligure, where he was doing time for a long series of thefts. Captured by the Germans after his escape and put on a train for the concentration camps, he was able to escape, but he was also forced to re-invent his life and give himself a new (false) identity. This document is a kind of autobiography of marginality found in the State Archive of Savona, in the Fondo Casa di Lavoro of Finale Ligure, where the petty criminal was sent again to serve his sentence until 1950, notwithstanding his fiery self-defence.

Tra il 1948 e il 1949 un lucidatore di mobili di Rieti, rinchiuso nelle carceri giudiziarie di Pordenone, scrive una particolare memoria autobiografica : un'autodifesa da presentare al locale Tribunale per respingere l'accusa di false generalità e appropriazione indebita. Reati - secondo il protagonista - indotti dalle circostanze che dopo l'8 settembre 1943 gli avevano consentito di evadere dalla Casa di lavoro di Finale Ligure, dove era rinchiuso per una lunga serie di furti. Catturato dai tedeschi dopo l'evasione e caricato su un treno diretto ai campi di concentramento, riuscirà a scappare, ma si troverà nella necessità di re-inventarsi una nuova vita ed una nuova/falsa identità. Il documento è una sorta di autobiografia della leggera ed è stato ritrovato presso l'Archivio di Stato di Savona, all'interno del Fondo Casa di lavoro di Finale Ligure, dove la leggera verrà rispedita per scontare la sua pena fino al 1950, nonostante l'agguerrita autodifesa.

\section{Storie imprigionate}

Il testo autobiografico preso qui in esame, trascritto integralmente, è conservato tra la documentazione della Casa di lavoro di Finale Ligure, depositata presso l'Archivio di Stato di Savona : 600 buste contengono i fascicoli personali dei detenuti e delle guardie di custodia, mentre 1338 fra registri matricole, agende contabili e volumi costituiscono la documentazione amministrativa $^{2}$. Si tratta quindi di una notevole mole di documenti, decine di migliaia di carte non an-

\footnotetext{
${ }^{1}$ Università degli Studi di Genova.

${ }^{2}$ Archivio di Stato di Savona, Casa di lavoro di Finale Ligure (1863-1965). Da ora ASS - Cdl.
} 
cora inventariate sistematicamente e studiate in modo organico. In effetti non è facile avvicinarsi a questo fondo, in quanto la disposizione dei documenti complica notevolmente eventuali ricerche : i fascicoli personali di centinaia di carcerati e del personale penitenziario sono raccolti alfabeticamente all'interno di ogni busta, che può così contenere materiali d'inizio Novecento $\mathrm{e}$ del Secondo dopoguerra ${ }^{3}$.

La Casa di lavoro ligure funzionò dal 1864 al 1965, mantenendo un rapporto sempre organico con il tessuto sociale cittadino : ciò a causa del particolare statuto della struttura che soprattutto nell'ultimo periodo di attività accoglieva detenuti al termine della condanna penale e prossimi al reinserimento sociale ${ }^{4}$. L'istituzione detentiva svolse un ruolo produttivo all'interno del paese, promuovendo attività professionali e l'acquisizione di specifiche competenze artigiane : a praticanti fabbri, sarti, calzolai, falegnami e tipografi venivano affidati durante la reclusione numerosi lavori, regolarmente retribuiti, e ciò creava un positivo indotto per l'intero tessuto economico locale ${ }^{5}$.

Un sondaggio dei documenti d'archivio, pur evidenziando lacune soprattutto per quanto riguarda la documentazione più antica, ha consentito di ricostruire aspetti della vita che si svolgeva all'interno del carcere e di ripercorrere le vicende, spesso travagliate, di alcuni dei numerosi ospiti. I fascicoli nominativi costituiscono in effetti una vera miniera di informazioni : dati anagrafici, caratteristiche e condizioni fisiche, annotazioni sul livello culturale, fedine penali, atti processuali, sentenze e talvolta anche le fotografie segnaletiche, non restituiscono solo la carriera criminale di molti individui, ma suggeriscono anche svariati percorsi di ricerca statistica, criminologica e sociale ${ }^{6}$.

\footnotetext{
${ }^{3}$ Cf. Fabio CAFFARENA, « Condenados a escribir », in Antonio CASTILLO GÓMEZ e Veronica SIERRA BLAS (dir.), Letras bajo sospecha, Gijón, Trea, 2005, pp. 107-133 (scaricabile in traduzione italiana dal sito www.balbisei.unige.it).

${ }^{4}$ Cf. Angela CAPELLI, La buona compagnia. Utopia e realtà carceraria nell'Italia del Risorgimento, Milano, Franco Angeli, 1988 e AA.VV., La Castiglia. Pagine di carcerazione dal regno di Sardegna ai giorni nostri (numero monografico di Il presente e la storia, 74, 2008).

${ }^{5}$ Cf. Michel FOUCAULT, Surveiller et punir. Naissance de la prison, Paris, Gallimard, 1975 (tr. it. di Alcesti TARCHETTI, Sorvegliare e punire. Nascita della prigione, Torino, Einaudi, 1993).

${ }^{6}$ Cf. Michel FOUCAULT, La vie des hommes infames, in Id., Dits et écrits, Paris, Gallimard, 1994, vol. III, pp. 237-255, (tr. it. di Graziella ZATTONI NESI, La vita degli uomini infami, Bologna, Il Mulino, 2009).
} 
In alcuni casi sono rimaste intrappolate nelle carte burocratiche anche numerose missive inviate e ricevute dai carcerati, per qualche ragione bloccate dalla censura dell'istituto : corrispondenze che in alcuni casi consentono di ricostruire, di esplorare in profondità storie di vita comuni ${ }^{7}$.

\section{Una leggera... anzi due}

Fra la documentazione giudiziaria custodita nel fascicolo di un detenuto è conservato un testo autografo del tutto atipico : si tratta di una memoria non datata scritta a scopo autodifensivo tra il 1948 e il 1949, rivolta direttamente alla Corte giudicante del Tribunale di Pordenone. L'autore della memoria difensiva è registrato come un lucidatore di mobili nato in provincia di Rieti nel maggio del 1914, povero, ma in possesso almeno della licenza di scuola elementare : potrebbe essere ancora vivo, circostanza che ne giustifica l'anonimato, così come quello degli altri protagonisti di questa storia. A ben vedere tale necessità sembra l'ironico epilogo per una vicenda di doppia identità, peraltro smascherata ${ }^{8}$.

Si trattava proprio di una leggera ${ }^{9}$ e per rendersene conto basta sfogliare la sua fitta carriera penale che copre gli anni dal 1930 - era appena sedicenne - al $1939^{10}$, quando dopo l'ennesimo furto viene dichiarato delinquente abituale e inviato alla casa di Lavoro di Apuania (Massa Carrara). Dopo qualche anno, anche la famiglia sembra scaricarlo :

Dopo oltre due anni - scrive il fratello da Livorno il 2 marzo 1942 - vengo di nuovo a farti rivedere il mio carattere perché essendo che mi trovo a compiere il mio sacro dovere da vero soldato Italiano come è stato il mio povero fratello [...]. [...] con oltre due anni non ti sei degnato di sapere le notizie del tuo dimenticato fratello [...] e finalmente ti sei degnato di scrivere a casa e di domandare di [...] e perché non ti scrive e non ti manda la sua foto. Oggi quando ho ricevuto la lettera da casa e mi ha detto di ciò che chiedi di me perciò mi sono deciso di scriverti e dirti ciò che mi sento da dirti. Egregio [...] mi sono deciso di scriverti per

\footnotetext{
${ }^{7}$ Le sole storie note sono quelle di Paolo VALERA, Dal cellulare a Finalborgo, Milano, Tipografia degli Operai, 1899 e Davide ALBERTARIO, Un Anno in Carcere : 2557, Milano, Ufficio dell'Osservatore cattolico, 1900.

${ }^{8}$ La memoria difensiva e gli altri documenti giudiziari citati si trovano in ASS - Cdl, busta 524

${ }^{9}$ Il riferimento è al lavoro di Danilo MONTALDI, Autobiografie della leggera, Torino, Einaudi, 1961.

${ }^{10}$ Dal certificato del Casellario giudiziale della Regia Procura di Rieti del 30 ottobre 1942 risultano una decina di condanne dal 1930 al 1939, soprattutto per furti.
} 
dirti delle raccomandazioni che ti feci come fratello lultima volta che ci siamo visti alla stazione di Orte che eri diretto per andare ad Aquila per lavorare e tutto invece ti sei recato a Ronciglione dove ai comesso un altro furto. E adesso ti rivolgi ai tuoi di casa dove all'età di 16 anni che manchi da casa per la tua testa sventurata e non ai dato altro che dispiaceri alla tua famiglia. Certo che io se mi sarei trovato alle condizioni tue e neanche avrei avuto il coraggio di ritornare ad scrivere a casa. Perciò ti voglio dire se chi è stato che ti a condotto a fare quessa disgraziata vita che da tutti viene odiata. Poi vorrei sapere se con che faccia che ti sei permesso di domandare di me.

Caro $[\ldots]$ perciò ti prego di non farti venire la voglia di scrivere più a casa e dimandare di me perché altrimenti neanche ti faccio sapere più niente di me e come di te non voglio più sapere neanche la puzza perché ne sono fino stufo di sentire dirmi che sei in galera e ogni volta che mi domandano di te mi vergogno perché essendo che io mi sendo di essere un vero cittadino. Perciò ti prego di dimenticarti addirittura di tuo fratello [...] devi far conto che non ce lai più perché tanto se per quando che sorti spero di ritrovarmi a casa anche io e perciò ti garantisco che se ritorni a casa ci sono io ti caccio da casa come se fossi un cane perché devi pensare che te a casa non ai dato un aiuto di nessuna maniera anzi ai cercato di sfruttare e di portare via perciò pensaci Prima di ritornare a casa e di essere sicuro che non ci sono io perché altrimenti se l'altra volta ti a caccia papà adesso ci sono io che comando a casa e neanche la porta ti faccio entrare. Per ora non mi prolungo altro ti saluto per eterno attendendo una tua risposta.

Mi firmo invano tuo

Dimenticabile fratello $[\ldots]$

Così, sul confine precario che passa fra rifiuto e accettazione, si chiude questa intensa missiva, in cui la paradossale attesa finale della risposta sembra un vero cortocircuito comunicativo fra la maldestra applicazione di una espressione formale e un'intima aspettativa.

In Liguria la leggera verrà trasferita l'8 marzo 1943 per scontare la pena fino all'8 marzo 1946: dopo 6 mesi dall'arrivo la sua vita sarà destinata ad incrociare la Grande Storia poichè l'armistizio italiano dell'8 settembre 1943, con la confusione e gli sbandamenti che seguiranno, lo mettono in condizione di evadere : 
[...] E mi porto col pensiero al punto di partenza - scrive nell'autodifesa - e cioè al lontano 10 settembre $1943^{11}$, in quel di Finale Ligure, tra le lugubri pareti di una casa di Lavoro.

Fu là che ebbe inizio la mia triste peregrinazione e fu in seguito agli eventi susseguitisi dopo 1'8 Settembre 1943 che io intravidi la possibilità di riformarmi una nuova vita.

Era notte quando in settecento circa $^{12}$, lasciammo la Casa di Lavoro e ci dileguammo attraverso la campagna ligure a piccoli gruppi, sparsi qua e là.

Ogn'uno di noi portava con sé un pesante fardello di anni di reclusione già scontati e con essi, le sofferenze patite ed accuitesi maggiormente in seguito al rigido tesseramento di guerra (erano i tempi di una minestra e di 150 grammi di pane al giorno ; pesavo $39 \mathrm{Kg}$. !) [...].

Tuttavia la fuga dal carcere ligure, avvenuta in seguito ad un bombardamento, si trasforma presto in un viaggio verso un ben più sinistro campo di concentramento nazista, a cui fortunatamente l'evaso riesce a sottrarsi grazie all'aiuto di alcuni abitanti di Pordenone, forse partigiani, che con la complicità di un militare austriaco rendono possibile la fuga dal treno.

L'inattesa liberazione rappresenta l'occasione per inventarsi una nuova esistenza assumendo il cognome materno per sottrarsi ad eventuali controlli : lavorerà come commerciante, aiutando la moglie nei mercati, ma la successiva nascita di una figlia, necessariamente registrata con il cognome falso, lo induce a rivelare la propria identità ed i propri precedenti penali alla compagna. Tradito dalla cognata, che non aveva mai accettato l'uomo, viene arrestato il 9 gennaio $1948^{13}$. A questo punto, dopo aver descritto minuziosamente gli eventi e la necessità di assumere una identità falsa, lo scrivente affronta direttamente gli aspetti giudiziari della causa in corso adducendo circostanziate motivazioni di forza maggiore per giustificare la propria condotta e rimettendosi infine alla clemenza del giudice.

Ma a destare interesse per il testo non è tanto l'aneddoto giudiziario, quanto la riscrittura di un'esperienza restituita attraverso una memoria scritta tutto sommato in modo corretto e ben articolata, che dosa sapientemente vittimismo, un'inaspettata competenza legale probabilmente instillata da un legale.

Il testo è incentrato sul principio dell'ineludibilità della scelta, del mutamento di identità visto come unica via d'uscita per salvare la vita. Attorno a questo principio fatale si costruisce l'intera

\footnotetext{
${ }^{11}$ I documenti presenti nel fascicolo riportano invece la data del 12 settembre 1943.

${ }^{12}$ Non è verosimile : un documento della Procura della Repubblica di Pordenone del 28 aprile 1948 attesta la fuga dal carcere ligure di ottanta persone.

${ }^{13}$ Così risulta dai documenti giudiziari : nella memoria si fa invece riferimento all'8 gennaio 1948.
} 
difesa, esempio pregnante della caleidoscopica natura delle scritture autobiografiche, che nel caso specifico miscela storia e storia di vita con le necessità processuali, nel tentativo di legittimare le proprie scelte. Tentativo peraltro fallito, poichè il Tribunale di Pordenone lo condannerà per il truffaldino cambio di nome. Rispedito nella Casa di lavoro finalese, sconterà la sua pena fino al 5 luglio 1950, nonostante il Giudice di Sorveglianza di Savona il 7 gennaio 1950 avesse riconosciuto che l'evasione fosse avvenuta per sottrarsi alla deportazione in Germania ${ }^{14}$.

Con l'apertura delle porte del carcere ligure si perdono le tracce della leggera, con in tasca il foglio di via per Pordenone: chissà se avrà potuto riabbracciare la figlia, la cui lontananza sarà sempre un vero e proprio cruccio. Lo dimostrano le numerose, accorate missive ai familiari rimaste imprigionate nel suo fascicolo, insieme a molte altre rivolte ai direttori dei carceri per lamentare il mancato pagamento di alcuni lavori, per richiedere di essere arruolato volontario durante la guerra, basta poter uscire..., ma anche per un taglio di capelli sbagliato dal barbiere.

\title{
3. L'autodifesa della leggera (1948/1949) ${ }^{15}$
}

\author{
Autodifesa dell'imputato $[\mathrm{A}][\mathrm{B}]^{16}$ \\ Ill.mo Signor Presidente, Signori della Corte !
}

Voi sapete ormai, attraverso l'incartamento processuale, di trovarvi di fronte ad uno dei tanti disgraziati casi della vita, casi che richiedono da parte Vostra molta comprensione e soprattutto molta umanità.

Io avrei preferito avere un difensore di fiducia, ma essendo privo di mezzi e quindi costretto a difendermi da solo, prego le S.V.Ill.me di voler seguire con benevolenza questa dolorosa dissertazione e di seguirmi attentamente fino in fondo.

\footnotetext{
${ }^{14}$ Cf. il riepilogo della situazione penale del condannato redatta a Finale Ligure in data 3 aprile 1952.

${ }^{15}$ Documento inedito.

${ }^{16}[\mathrm{~A}]$ e $[\mathrm{B}]$ indicano rispettivamente cognome e nome reali dell'imputato. [C] e [D] cognome e nome assunti dopo il cambio di identità. Le altre lettere dell'alfabeto, dalla [E] alla [I], i nomi delle persone rese anonime per motivi di riservatezza.
} 


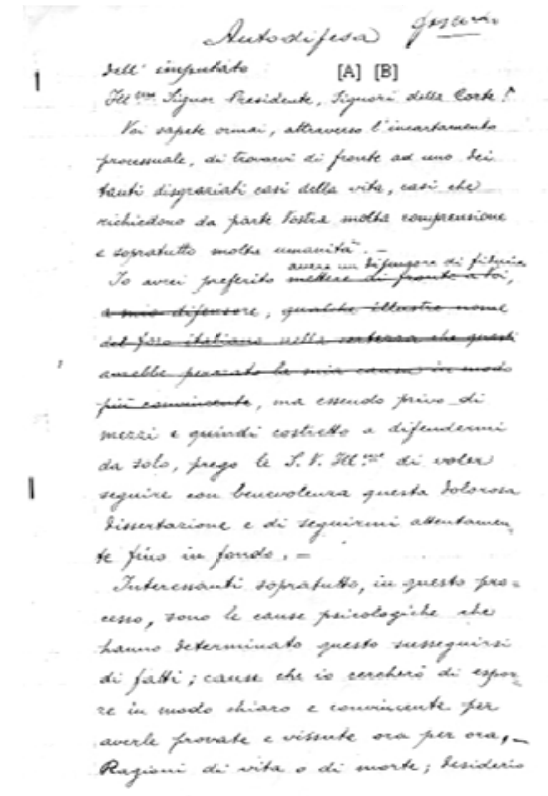

Interessanti soprattutto, in questo processo, sono le cause psicologiche che hanno determinato questo susseguirsi di fatti ; cause che io cercherò di esporre in modo chiaro e convincente per averle provate e vissute ora per ora. Ragioni di vita o di morte ; desiderio di redenzione; desiderio di rifarmi una nuova esistenza sotto l'auspicio di uno dei più sacri sentimenti umani, ch'io ho sempre tenuto in alto: la formazione di una famiglia che mi facesse dimenticare un doloroso passato e mi dasse la forza di ben proseguire nell'avvenire. E mi porto col pensiero al punto di partenza e cioè al lontano 10 Settembre 1943, in quel di Finale Ligure, tra le lugubre pareti di una Casa di Lavoro.

$\mathrm{Fu}$ là che ebbe inizio la mia triste peregrinazione e fu in seguito agli eventi susseguitisi dopo l'8 Settembre 1943 che io intravidi la possibilità di rifarmi una nuova vita.

Era notte quando in settecento circa, lasciammo la Casa di Lavoro e ci dileguammo attraverso la campagna ligure a piccoli gruppi, sparsi quà e là.

Ogn'uno di noi portava con sè un pesante fardello di anni di reclusione già scontati e con essi, le sofferenze patite ed acuitesi maggiormente in seguito al rigido tesseramento di seguito guerra (erano i tempi di una minestra e di 150 grammi di pane al giorno ; pesavo $39 \mathrm{Kg}$. !).

Fuori c'era il caos. Proseguii solo, deciso ad affrontare i disagi ed i pericoli pur di raggiungere la mia famiglia nei pressi di Roma ; ma dopo dieci giorni di vane peregrinazioni, giunto a Bologna, fui fermato dai tedeschi e caricato su un treno merci dove altre centinaia di persone attendevano inconscie la loro deportazione in Germania.

Addio sogni e vane illusioni ; addio speranze !

Tutto era crollato.

Partimmo da Bologna il 20 Settembre stipati in carri bestiame (50, 60 persone per carro, in piedi) e per di più ermeticamente chiusi e sigillate le porte. Quattro giorni e quattro notti di 
viaggio, senza mangiare, senza bere, senza possibilità di soddisfare i bisogni corporali che in vagone ; ed arrivammo, in quelle condizioni, a Pordenone.

Questa simpatica cittadina aveva già organizzato $\mathrm{i}$ primi soccorsi in nostro favore e predisposto, prima che il convoglio si rimettesse in moto (con la complicità della scorta austriaca), che fossero tolti i sigilli ed i chiavistelli alle porte e che fosse così favorita la nostra fuga in massa che doveva avvenire ad un rallentamento del treno già disposto nei pressi di Cusano. E così facemmo.

Ripresa la libertà e braccati continuamente dalle S.S. germaniche, ci siamo dati tutti alla macchia e poi raggiungemmo Pordenone chiedendo asilo e nascondiglio nelle famiglie private. Si trattava di vita o di morte ; i tedeschi, in simili frangenti, non risparmiavano nessuno ; io, poi avevo anche ragione di temere di essere ricercato dalla Polizia italiana per l'evasione in massa dalla Casa di Lavoro di Finale Ligure avvenuta quattordici giorni prima, in seguito a violento bombardamento.

Fu così che, per evitare guai, assunsi il nome di [C] [D] (cognome di mia madre) e mi misi a disposizione del locale Comitato della Croce Rossa, presieduto dal Dott. Brunetta e dal Cav. Toffoli, come sbandato proveniente dall'areonautica.

Da una crocerossina fui accompagnato nella Famiglia $[E]$ dove ebbi ospitalità e dove conobbi $\mathrm{l}^{\prime}[\mathrm{E}][\mathrm{F}]$ divenuta in seguito mia moglie.

Ecco, o Signori della Corte, quale fu la causa principale che ha determinato il cambio delle mie generalità e da dove è partito il filo conduttore di questa aggrovigliata matassa.

Voi siete dei padri di famiglia ; qualcuno di Voi avrà forse sofferto per i figli le stesse ansie, gli stessi dolori, le medesime angoscie di tanti altri padri e certamente avrete benedetto coloro che si sono adoperati in tutti i modi e con tutti i mezzi per salvare loro la vita. Tutti Voi conoscete le dolorose conseguenze delle deportazioni in germania e nessuno di Voi, ancor oggi, riterrà una infrazione alla legge tutto ciò che si è fatto allora, consenzienti le stesse Autorità, per risparmiare tante vite umane.

E tutti voi, infine, siete a conoscenza che proprio dai fuori legge di allora, o almeno considerati tali dagli avversari, nacque l'ormai famoso movimento di liberazione nazionale che tanto diede alla Patria.

Ho ritenuto opportuno descrivervi prima l'ambiente e poi fissare definitivamente ed in modo inequivocabile il punto di appoggio su cui necessariamente si basa questa mia autodifesa.

Come dissi, a Pordenone trovai asilo presso la Famiglia [E] e là ricominciai la mia nuova esistenza. 
La signora $[\mathrm{F}]$ faceva i mercati come ambulante ed io, alternando il servizio con la Croce Rossa in Stazione e a tempo perso, cercavo di rendermi utile sia in casa che fuori perchè non volevo assolutamente gravare sul bilancio famigliare.

Ebbi così modo di esplicare la mia attività anche sul campo commerciale e di cattivarmi la stima e la simpatia della Signora che in seguito mi incoraggiò a proseguire e si adoperò per farmi avere le relative licenze di commercio.

Non mi dilungo in particolari che potrebbero annoiarVi, Vi dico soltanto che sono partito con tremila lire, frutto di una colletta fra amici e poco a poco, facendo i mercati prima ed arrischiando viaggi su viaggi a Milano poi, sotto bombardamenti, disagi di ogni specie dovuti a mancanza di trasporti ed ai continui pericoli di perdere la merce o di vedersela confiscata per istrada, riuscii in pochi mesi a crearmi una base finanziaria abbastanza solida per poter continuare il mio commercio da solo, senza preoccupazioni di sorta.

Ebbi incarichi di ogni genere dai negozianti di Pordenone ai quali provvedevo la merce a Milano, Como, Bergamo, Biella, Venezia ecc. ; ebbi in consegna somme rilevantissime destinate all'uopo e feci sempre buona figura con tutti, rendendo conto a tutti fino all'ultimo centesimo e rinsaldando nel contempo la mia posizione finanziaria che si è andata facendo invidiabile con la stima e la benevolenza di tutti.

$\mathrm{Fu}$ naturalmente all'inizio di questa mia fortunata carriera che nacquero i primi sentimenti di simpatia reciproca con la Signora $[\mathrm{E}][\mathrm{F}]$ ved. $[\mathrm{G}]$, sentimenti di riconoscenza in un primo tempo e tramutatisi, col passar dei giorni, in amore e travolgente passione.

Non è stata la differenza di età (Lei era dieci anni più vecchia di me); non c'è stato l'ostacolo di un figlio di sedici anni, che Lei aveva avuto col rimo marito, a frenare questo mio impulso di cuore, impulso naturale dovuto alla fiducia cieca nell'avvenire, che si prospettava roseo ed al desiderio di sistemare la mia vita in modo leale e definitivo, dimenticando un passato di dolore.

Mi buttai a capofitto nell'avventura, arrischiando tutto per tutto, ma sempre sorretto da sentimenti buoni, da principi onesti e dal desiderio di riabilitarmi ; e vi riuscii.

In questo periodo, le relazioni con colei che doveva poi diventare la compagna della mia vita, divennero intime ; i miei interessi erano i suoi ; la cassa era unica e lavoravamo entrambi per raggiungere un unico scopo : la creazione di una nuova famiglia.

Nel Giugno 1944 le feci smettere di fare i mercati, accollandomi tutto il peso della numerosa famiglia.

L'unica sorpresa spiacevole mi venne dalla sorella di Lei e nome $[\mathrm{H}]$ che, all'insaputa di tutti, nel Luglio 1944, mi denunciò al Comando Tedesco ed alla Questura di Pordenone come capo 
partigiano operante nella zona di Conegliano, il che non era vero, non avendo mai appartenuto a nessuna organizzazione nè partigiana, nè fascista.

Ebbi dei grattacapi, fui trattenuto in Questura e da Leo Massa e dal Capitano Vettorini, che mi conoscevano, fui rimesso in libertà e da loro stessi venni informato che la denuncia era stata fatta e firmata dalla futura cognata ; notizia, d'altra parte, che mi è stata confermata, in seguito, dalla Signorina Irma Bottega di Cordenons.

Non ho mai saputo perchè $l^{\prime}[\mathrm{H}]$ avesse fatto questo e il perchè nutrisse tanto livore nei miei riguardi.

Non dissi niente a casa, ma sapevo ormai di avere, in casa, una nemica che dovevo temere.

Ripresi la mia attività ; tutto andava di bene in meglio quando, nell'Ottobre dello stesso anno, la mia futura moglie rimase incinta.

Sorpresa generale in famiglia ; paura di scandali ; primi attriti fra me e la sorella di Lei, [H], che non vedeva di buon occhio neanche questa nostra relazione ; intervento dei cognati per sistemare ogni cosa e finalmente la proposta di matrimonio.

Eravamo, come ripeto, nel 1944 e per me i pericoli non erano ancora scomparsi. Fuori tutto continuava come prima : persecuzioni, angherie, sopprusi, deportazioni ecc. che cosa dovevo fare?

Quì, o Signori, scrivo forse uno dei capitoli più importanti della mia vita. Soverchiato dagli eventi, ma sorretto dalla Fede e da un profondo sentimento umano, presi decisamente la mia strada e affrontai senza debolezze il mio destino.

Avrei potuto riprendere il mio capitale e andarmene insalutato ospite, lasciando una madre incinta in balia di sè stessa ; avrei potuto lasciare una famiglia, dalla quale non avevo avuto che bene, nella disperazione e nel disonore : avrei potuto, se fossi stato un malvagio, piantare tutto e tutti, ed invece non lo feci, anzi, affrontai la situazione a core aperto.

L'unica proposta, dettatami dalla coscienza, fu quella di protrarre il matrimonio fino al dopo guerra dando ai famigliari le più ampie garanzie sulla serietà dei miei propositi ; ma, anche questa, cadde di fronte alla situazione ed alle pressioni d'indole morale dei parenti ; ed il 3 Dicembre 1944, mi sposai. Dal matrimonio [C]-[E] nacque, il 16 Luglio 1945, la piccola [I] che naturalmente venne registrata, allo Stato Civile di Pordenone, sotto il nome di Marini.

E quì, entriamo nel vivo della causa. Potevo io, [A], dopo aver contratto regolare matrimonio col nome di [C], registrare la neonata sotto altro nome che non fosse quello di [C] ? No, perchè di fronte alla legge avrei commesso un falso. Sembra un paradosso, ma è così, e mi spiego : astraendo dal fatto ch'io fossi realmente [A], risulta però evidente che nessuno mi conosceva come tale e, sia allo Stato Civile di Pordenone, che nella vita privata, ero registrato e 
conosciuto per $[\mathrm{C}]$ e quindi, come tale, non potevo certamente nè esimermi dall'obbligo di denunciare la nascita della piccola allo Stato Civile, nè tantomeno di denunciarla sotto altro nome. Questo sdoppiamento di persona avrebbe comportato, come premessa, una rettifica del nome, fatta a tempo debito, rettifica che in quell'epoca non poteva ancora esser fatta per le note ragioni, ma che era nelle mie intenzioni, ben sapendo che se non l'avessi fatta di mia iniziativa, tosto o tardi vi sarei stato costretto da qualche evento impreveduto.

Alla fine dei conti, che cos'era che mi impediva di farlo ?

Niente, o per lo meno, ragioni superabilissime ; una volta provate le cause che mi costrinsero al cambio delle generalità, non rimaneva che mettere al corrente mia moglie dell'accaduto e mettermi a disposizione delle Autorità per la rimanenza della Casa di Lavoro. Tanto più che per quest'ultima, data la buona condotta tenuta nel frattempo e giustificate le cause dell'evasione, potevo ancora sperare, con l'aiuto del Giudice di Sorveglianza, di scansarne la rimanente esecuzione, trattandosi di una misura di sicurezza detentiva per la quale il Giudice stesso poteva chiederne la sospensione, se concorrevano i requisiti necessari.

Otto mesi di casa di Lavoro, li avevo ormai scontati.

A questo punto viene spontanea da parte Vostra la domanda : "perchè non l'avete fatto ?" È giusto ; questo fu il mio errore e non vi trovo molte giustificazioni.

Ho dormito troppo sugli allori ; ho aspettato, necessariamente, la fine della guerra ; sono rimasto, poi, in attesa di vedere come si mettevano le cose ed infine, ho rimandato di giorno in giorno la decisione finale dicendo a me stesso che quando volevo farlo ero sempre in tempo e che frattanto avrei preparato con calma il terreno propizio. Il passo era duro e richiedeva molta meditazione ; d'altra parte, un primo passo lo avevo già fatto coll'informare mia moglie di tutto, anche dei miei precedenti penali.

Posso aggiungere, anzi, che fu proprio in seguito alla confessione fatta a mia moglie che la cognata $[\mathrm{H}]$, venuta a conoscenza della cosa, non so come, se ne fece un'arma per colpirmi al momento opportuno, a mezzo di terze persone, provocando il mio arresto (avvenuto a Vittorio Veneto l'8 Gennaio 1948) e la conseguente rottura dei rapporti con mia moglie, causa delle mie miserevoli condizioni attuali.

Non faccio addebiti di alcun genere nè a mia moglie, nè a nessuno, per questo ; affermo soltanto che ho la coscienza tranquilla di aver fatto il mio dovere di marito e di padre e di aver lasciato una famiglia in grado di continuare, nel lavoro, una vita scevra da preoccupazioni di sorta. 
Ritornando quindi al falso della piccola, che mi ha portato in Assise, mi permetto di far osservare all'Eccellentissima Corte che questo reato non può essere considerato come reato a sè e tanto meno può essere considerato reato, per mancanza di dolo.

Non può essere considerato come reato a sè, perchè parte integrante e conseguenza inevitabile di un unico falso precedente. Il [C], come tale, non ha commesso nessun falso. Il [C], ad esempio, se al momento della notificazione della nascita della figlia allo Stato Civile, avesse chiesto di iscriverla all'anagrafe sotto il nome di [A], gli avrebbero riso in faccia e risposto che non poteva farlo perchè avrebbe commesso un falso. Dov'è dunque questo falso della piccola, preso in se stesso, che l'atto di accusa sostiene?

Esiste sì un falso, ma è sempre lo stesso falso commesso dal [A] fin dall'inizio e cioè fin dal momento che è stato costretto a chiamarsi [C] [D]. Tutto il resto è una continuazione di falso e niente più.

Non può essere considerato come reato perchè, se l'articolo 567 del Codice Penale commina una pena gravissima che va da 5 a15 anni di reclusione lo fa per punire coloro che provocano le conseguenze dolose di un tale reato.

Quì non esistono conseguenze dolose. La registrazione della piccola non è stata fatta per occultare una nascita illegittima, ma bensì per legittimare una creatura frutto naturale dell'unione regolare di due coniugi che l'hanno sempre adorata e tenuta con sè come un angelo vivificatore.

Non vi è abbandono di neonato e tantomeno esistono altre cause occulte.

Tutto è chiaro e tutto è stato fatto alla luce del sole.

Se non è stato possibile fare altrimenti, le ragioni le conoscete già, come già siete a conoscenza che tosto o tardi la rettifica sarebbe avvenuta da sè essendo la cosa ormai palese e quindi inevitabile.

Chiarito questo punto essenziale della causa, non mi rimane che il primo falso, come causa determinante.

Se ho cercato, fin dall'inizio, di farvi conoscere il quadro della situazione e se ho cercato di illustrarVi, nel miglior modo possibile, sia l'ambiente ch le cause che hanno determinato il cambio delle mie generalità, non l'ho fatto a caso.

Esiste una causa di forza maggiore che io, se mi sarà concesso, cercherò di far valere invocando, dall'Eccellentisima Corte, l'applicazione dell'Art. 54 del C.P. che dice : « non è punibile chi ha commesso il fatto per esservi stato costretto dalla necessità di salvare sè od altri dal pericolo attuale di un danno grave alla persona, pericolo da lui non volontariamente causato, nè altrimenti evitabile, sempre che il fatto sia proporzionato al pericolo ». 
Non ritengo opportuno fare commenti ; l'articolo del Codice è chiaro ed entra da sè nel vivo della causa.

Nessuno potrà mai negare che la fuga dal treno di deportazione in Germania, avvenuta a Pordenone il 24 Settembre 1943 e di cui feci parte, abbia costretto me ed altri a vivere sotto altro nome per non essere scoperti e per salvare la nostra vita. Nessuno potrà mai dire che $\mathrm{i}$ fatti succedutisi in seguito e di cui fui l'attore, siano stati sproporzionati al pericolo. Quindi, gli estremi per l'applicazione del suddetto articolo sono stati raggiunti, ed io, alla benevolenza dell'Eccellentissima Corte, non chiedo altro.

Chiudo questa mia autodifesa invocando un giudizio sereno. Sia tenuto conto delle cause di forza maggiore che hanno causato gli eventi ; sia tenuto nel debito conto la buona intenzione di raggiungere uno scopo a fin di bene ; sia dato ai fatti il valore che essi meritano, ed infine, se mi credete meritevole di una condanna, condannatemi per la non avvenuta rettifica delle mie generalità e cioè per quello che realmente dovevo fare e non ho fatto a tempo opportuno.

Ill.mo Signor Presidente, Signori della Corte ! ho trascorso ormai... mesi di carcere in attesa di questo Vostro verdetto, privato dell'affetto dei miei cari, fate almeno che esso sia mite e tale da restituire un padre all'affetto della propria creatura e gli sia di sprone per incamminarsi nuovamente sulla retta via. 